\title{
Imaging of pulmonary infections after lung transplantation: a pictorial essay of early and late computed tomography findings
}

\author{
Chiara Giraudo ${ }^{1}$ - Giulia Rizzon ${ }^{1} \cdot$ Linda Mazzai $^{1} \cdot$ Monica Loy ${ }^{2} \cdot$ Elisabetta Balestro $^{3} \cdot$ Raffaella Motta ${ }^{1}$. \\ Federica Pezzuto ${ }^{4} \cdot$ Roberta Polverosi $^{5} \cdot$ Fiorella Calabrese $^{4} \cdot$ Federico Rea $^{2}$
}

Received: 9 October 2021 / Accepted: 10 January 2022 / Published online: 24 January 2022

(c) The Author(s) 2022

\begin{abstract}
Pulmonary infections are among the most common complications after lung transplants and a major cause of morbidity and mortality in these patients. Computed tomography is one of the main non-invasive diagnostic tools for detecting lung infections but characterizing the correct etiology may be very challenging. Indeed, although several pathogens show typical patterns at imaging, others, such as bacteria, may demonstrate quite unspecific features. Therefore, additional parameters, like the timing of the infection, should be evaluated to support the radiologists in narrowing the differential diagnoses. In fact, it has been demonstrated that several pathogens, like Candida albicans, usually occurring within the first month after the transplant, frequently occur at specific time points. Thus, aim of this review is to make radiologists and clinicians familiar with the computed tomography patterns of pulmonary infections occurring after lung transplant, considering the etiology and the time of onset, according to the extensive experience gained in our tertiary center.
\end{abstract}

Keywords Computed tomography $\cdot$ Pulmonary infections $\cdot$ Lung transplant

\section{Introduction}

Pulmonary infections are a leading cause of morbidity and mortality after lung transplant (LT) and may occur at any time [1-4]. LTs carry an intrinsic high risk of infection because of the direct communication of the transplanted organ with the atmosphere [5]. Additional factors associated with the surgical procedures (e.g., impaired mucociliary function due to allograft denervation, administration of immunosuppressive drug therapy) [6], the donor (e.g., insufficient donor arterial oxygen tension, positive donor sputum)

Chiara Giraudo

chiara.giraudo@unipd.it

1 Department of Medicine - DIMED, University of Padova, Via Giustiniani 2, 35100 Padova, Italy

2 Thoracic Surgery Unit, Department of Cardio-Thoracic and Vascular Sciences, University of Padova, Padova, Italy

3 Respiratory Disease Unit, Department of Cardio-Thoracic and Vascular Sciences, University of Padova, Padova, Italy

4 Pathology Unit, Department of Cardio-Thoracic and Vascular Sciences, University of Padova, Padova, Italy

5 Istituto Diagnostico Antoniano - Affidea, Padova, Italy and the recipient (e.g., older than 40 years) $[1,6]$ may play a significant role for the onset of infections in the early and late postoperative phase.

Together with the clinical and laboratory evaluations, radiological imaging, especially computed tomography (CT), is crucial for the early detection and characterization of pulmonary infections in LT [1-4]. Indeed, specific patterns at $\mathrm{CT}$ such as nodules with a peripheral rim of groundglass due to Aspergillosis [1,7] or diffuse bilateral groundglass areas with sub-pleural sparing due to Pneumocystis jirovecii may suggest a confident diagnosis. Even if in some cases, such as with bacterial infections, the imaging features might be relatively unspecific, making the diagnostic process more challenging [1-3], the temporal relationship between the LT and the onset of the lesions may narrow down the list of differential diagnoses. In fact, it is well known that specific agents, such as the Pseudomonas aeruginosa, which is more frequent during the early postoperative phase, tend to occur at specific time points.

Thus, the aim of this review is to make radiologists and clinicians familiar with the $\mathrm{CT}$ patterns pulmonary infections occurring after LT considering the etiology and the time of onset of the disease. 


\section{Pulmonary infections after lung transplant}

Since different agents are associated with the occurrence of pulmonary infections at different time points after LT, a subdivision in early (up to 1 month), primary late (1-4 months), and secondary late (after 4 months) infections, according also to the different etiologies, has been applied (Fig. 1) [1, 2].

\section{Early infections (up to 1 month)}

\section{Bacterial infections}

Bacterial infections are the most common postoperative infections in LT recipients, especially during the early phase (i.e., 1 month). Nevertheless, it should be considered that they may also occur later on during recipients' life $[8,9]$.

Gram-negative bacilli, such as Klebsiella species and Pseudomonas aeruginosa, followed by Gram-positive, like Staphylococcus aureus, are the most frequent agents [6]. Pulmonary Actinomycosis, even if rare, should be considered among the differential diagnoses especially in case of mediastinitis [10]. It has also to be highlighted that recipients with cystic fibrosis carry a higher risk of infections due to Pseudomonas aeruginosa and Burkholderia cepacia complex.

Unfortunately, the CT features of bacterial infections are quite unspecific, since they may include atelectasis, bronchocentric opacities, airspace consolidations (i.e., sub-segmental, segmental, or lobar), branching nodular and linear opacities (i.e., tree-in-bud), interlobular septal thickening, and pleural effusions [1] (Figs. 2 and 3). Pulmonary Actinomycosis may cause areas of consolidation surrounded by ground-glass opacities (Fig. 4). Staphylococcal pneumonia is frequently complicated by the development of pneumatoceles (Fig. 5).

\section{Fungal infection}

Candida species often colonize the airways of LT recipients. Very aggressive infections usually occur during the first month after the LT, either as postoperative complications due to a prolonged stay in the intensive care unit or because of a colonized donor organ [11-13].

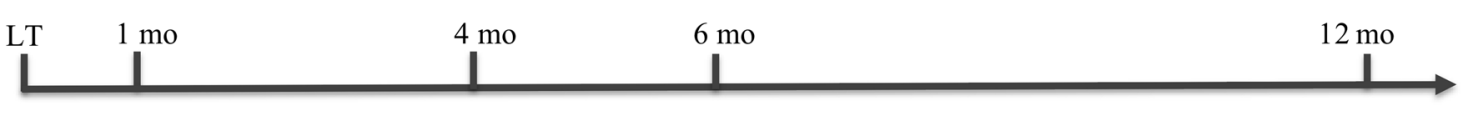

Bacteria $^{\mathrm{a}}$

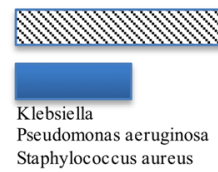

Staphylococcus aureus

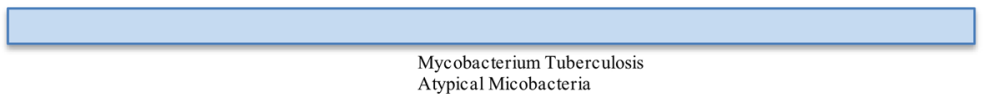

Fungi

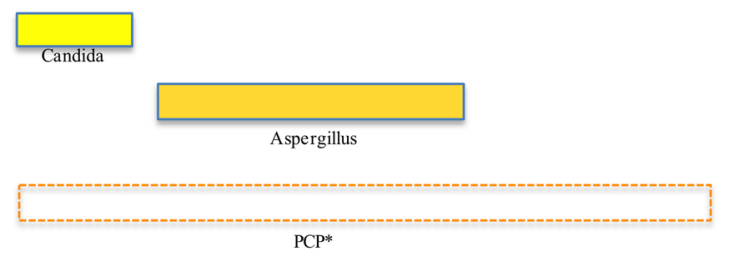

Atypical Micobacteria

Viruses $^{b}$

Notes: LT= lung transplant; mo= month/s; $\mathrm{PCP}=$ Pneumocystis Carinii; $\mathrm{CMV}=$ Citomegalovirus; $*$ = period of occurrence of the infection of Pneumocystis Carinii prior to the trimethoprim/sulfamethoxazol prophylaxis

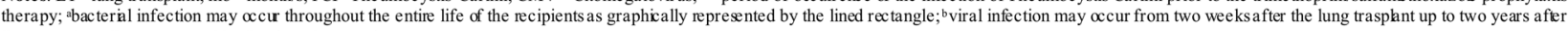
it, as graphycally represented by the dotted rectangle.

Fig. 1 Graphic representation of the temporal distribution of the main agents causing pulmonary infections after lung transplant [1,2] 


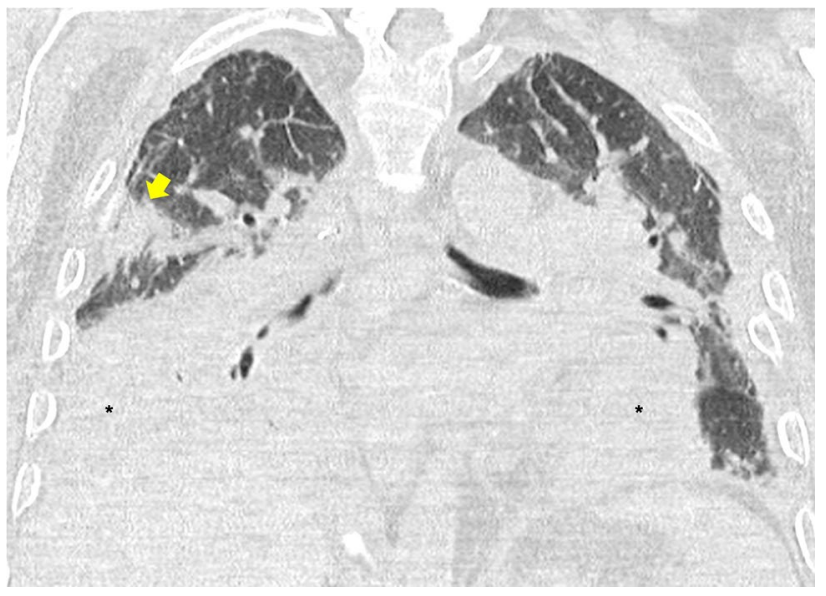

Fig. 2 Multidrug-resistant Klebsiella infection in a 56-year-old man who underwent bilateral lung transplant for pulmonary emphysema 27 days before. The coronal reconstruction of the computed tomography scan demonstrated typical bacterial findings represented by pulmonary segmental and lobar consolidations respectively in the middle (yellow arrow) and lower lobes (black asterisks)

CT patterns of fungal infections include patchy and confluent pulmonary infiltrates, nodules (i.e., occasionally miliary), mass-like foci or airspace consolidation, and interstitial involvement. In case of single LTs, fungal pneumonia mainly affects the transplanted lung (Fig. 6). Very aggressive infections may also cause candidemia, mediastinitis, or necrosis of the anastomosis [8].

\section{Primary late infections (1-4 months)}

\section{Viral infections}

Respiratory viral infections are a very important cause of morbidity and mortality in LT recipients [14].
Cytomegalovirus (CMV) is the second-most common cause of pneumonia and the most common opportunistic infection in LT patients $[1,15]$, even if it has to be considered that the current prophylaxis with antiviral drugs is reducing its occurrence [16]. Overall, CMV pulmonary infections have a peak incidence at $1-4$ months (i.e., range 1-12 months) rarely occurring in the first two weeks after the LT [1]. Primary pulmonary infections due to CMV are usually very severe and may occur in seronegative recipients receiving a graft from a seropositive donor. On the contrary, secondary infections, commonly less severe, are mainly diagnosed in seropositive recipients either infected by a different CMV strain or undergoing a reactivation due to immunosuppression [8].

In addition to CMV, community-acquired viruses, such as respiratory syncytial, parainfluenza, influenza virus, and adenovirus, may infect LT recipients especially between 2 weeks and 2 years after the LT, with a rate of occurrence between 8 and $14 \%$ [1].

Other less common viral pathogens are herpes simplex (i.e., the incidence is constantly decreasing because of prophylaxis), varicella zoster (i.e., mainly causing muco-cutaneous involvement), and Epstein-Barr virus (i.e., especially important because it may then cause lymphoproliferative disorders) [8].

CT findings of viral infections include ground-glass, air-space consolidations, tree-in-bud opacities, airway dilatation, bronchial wall thickening, and pleural effusion (Fig. 7) [1, 8, 17]. Severe infections may present with a crazy paving pattern (i.e., ground-glass opacity with superimposed interlobular septal thickening and intra-lobular reticular thickening) (Fig. 8). The CMV pneumonia is also associated with a high risk of superimposed bacterial and fungal infections. Thus, it needs to be taken into consideration that findings due to the additional infection

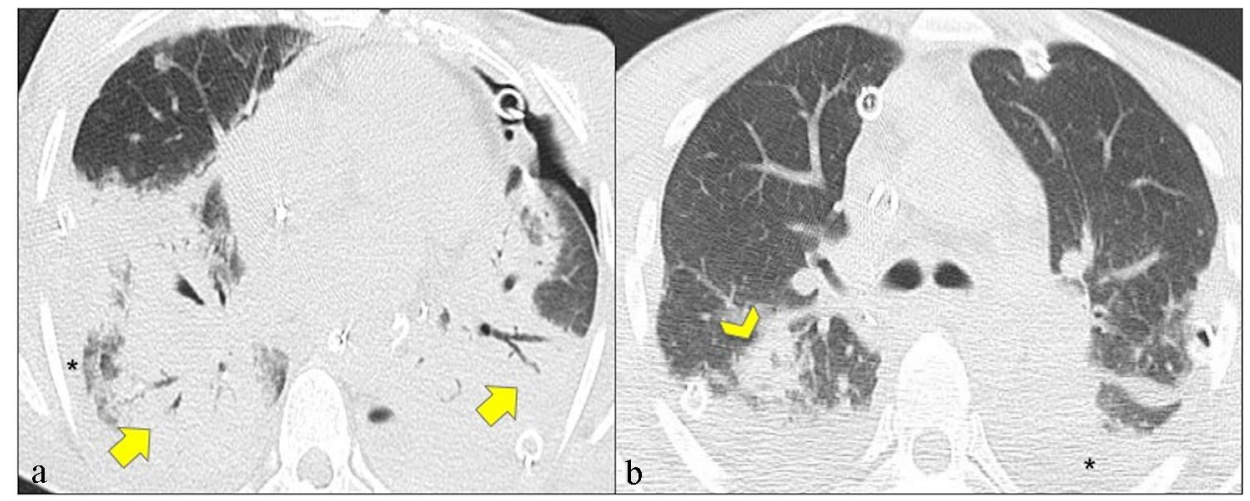

Fig. 3 Two patients (31 and 24 years old man and woman, respectively in $\mathbf{a}$ and $\mathbf{b}$ ) affected by bacterial pneumonia after bilateral lung transplant for cystic fibrosis (i.e., one month before). The patient was affected by Pseudomonas aeruginosa and showed pulmonary consoli- dations with air bronchogram (yellow arrows in a). The patient in b had a pulmonary round consolidation (yellow arrowhead in b) due to Burkholderia cepacia. Both patients showed also pleural effusion (black asterisks in $\mathbf{a}$ and $\mathbf{b}$ ) 


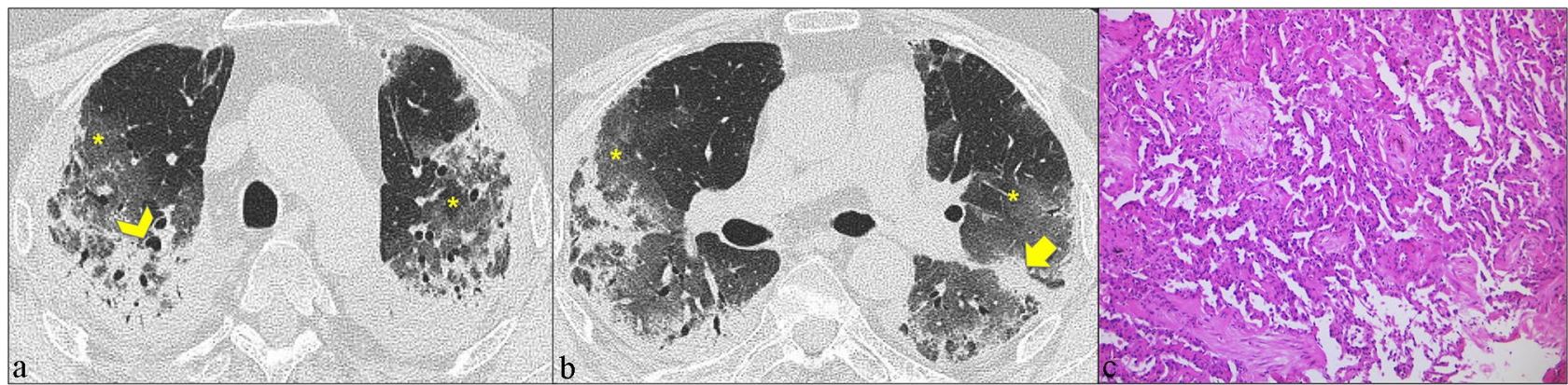

Fig. 4 48-year-old man affected by Actinomyces pneumonia one month after bilateral lung transplant. This rare agent caused multifocal areas of consolidation and ground-glass (yellow asterisks in a and $\mathbf{b}$ ) as well as bronchiectasis (yellow arrowhead in a), and thickening of the major left fissure (yellow arrow in b) easily detected at computed tomography. The transbronchial biopsy showed interstitial fibrosis with several Masson body. The final histological diagnosis was organizing pneumonia, the histological pattern may recognize different etiology, including infections (hematoxylin and eosin, original magnification $\times 100$ )

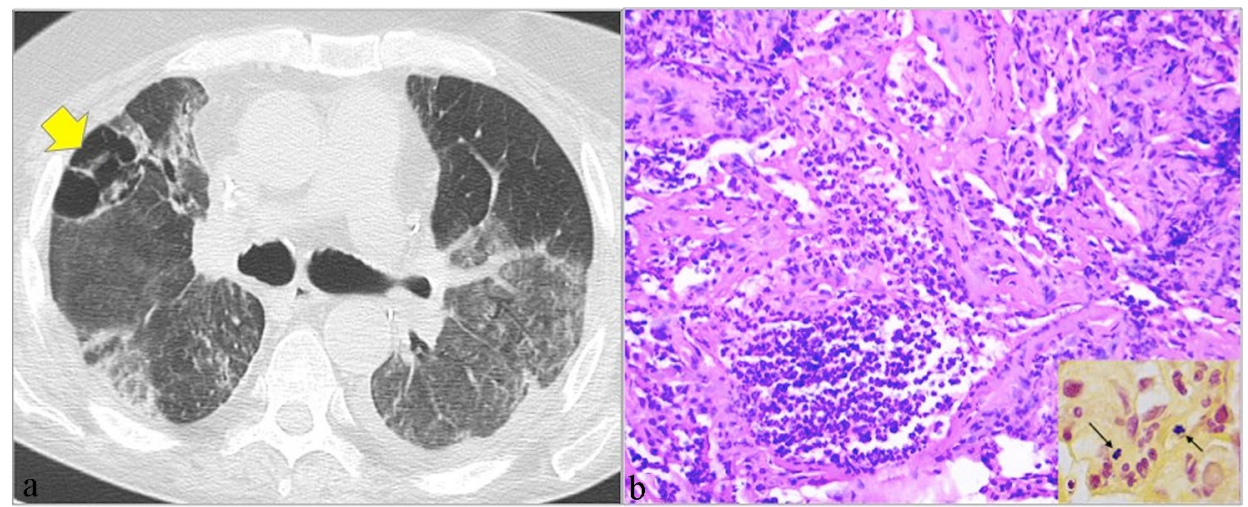

Fig. 5 55-year-old man affected by Staphylococcus aureus pneumonia, which occurred three months after the bilateral LT for idiopathic pulmonary fibrosis. The chest CT revealed a pneumatocele (yellow arrow in a) which is a typical complication of pulmonary infections due to this bacterium. The transbronchial biopsy showed a massive

could overlap with the above-mentioned viral features at imaging (Fig. 9).

\section{Fungal infection}

Aspergillosis is the most common fungal infection in LT recipients $[7,18]$ and it usually occurs $1-6$ months after the LT (i.e., peak incidence within the first 3 months after the LT) [1].

Ulcerative tracheobronchitis is the earliest and most common expression of the disease, and it may cause anastomotic interstitial and endoalveolar neutrophilic infiltration (hematoxylin and eosin, original magnification $\times 200$ in b). GRAM stain showed aggregate of positive bacteria (arrows in c) (inset: GRAM stain, original magnification $\times 600$ )

dehiscence. Its diagnosis is easily reached at CT with the detection of focal bronchial wall defects and perianastomotic air collections (Fig. 10) [19].

Aspergilloma, necrotizing pneumonia, invasive pulmonary disease, disseminated infection, or empyema may also be due to Aspergillus infection. Invasive pulmonary infections tend to occur in the later phase after LT and are mainly associated with the dissemination to other organs. Typical features at CT imaging of Aspergillus infection include focal nodular and mass-like consolidations, cavitation, nodules (i.e., solitary or multiple) surrounded by a rim of 


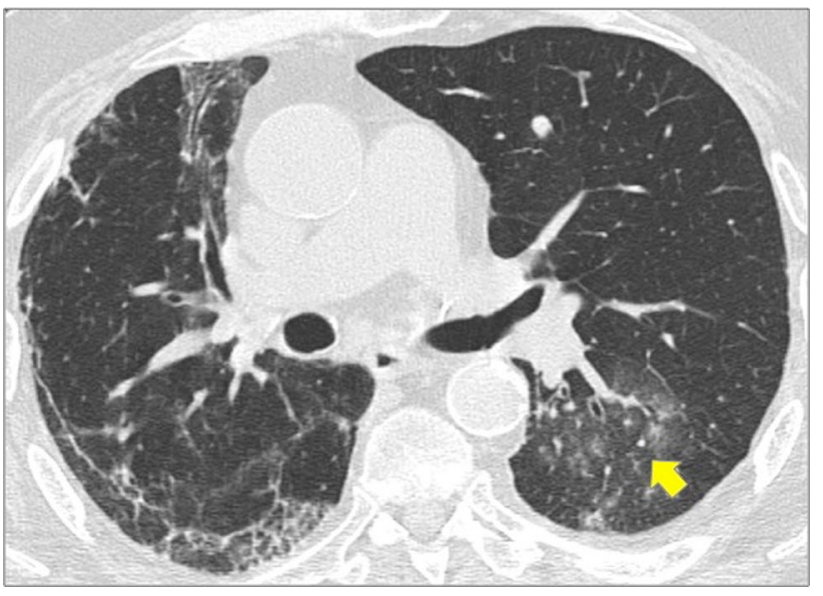

Fig. 6 Candida albicans infection in a 67-year-old man, who underwent a single, left LT for idiopathic pulmonary fibrosis 20 days before. Axial high-resolution computed tomography image showing micro-nodules, ground-glass and airspace opacities affecting the transplanted lung (yellow arrow); fibrotic changes due to interstitial pneumonia are easily detected in the right lung ground-glass opacity (i.e., halo sign), and pleural thickening (Figs. 11 and 12) [1, 7].

\section{Secondary late infections (> 4 months)}

\section{Bacteria}

Among the bacterial infections occurring in the secondary late period after LT, those due to Mycobacterium tuberculosis are quite frequent (i.e., estimated prevalence between 2 and $3.8 \%$ ) [1, 8], and are usually caused by the reactivation of a focus in the native lung or by transmission via the allograft.

The colonization by atypical mycobacteria occurs also fairly frequently (0.46-8\%) [19-21] with Mycobacterium abscessus complex being one of the main agents [15].

CT findings of typical and atypical species include clusters of multiple small nodules, ground-glass opacities or

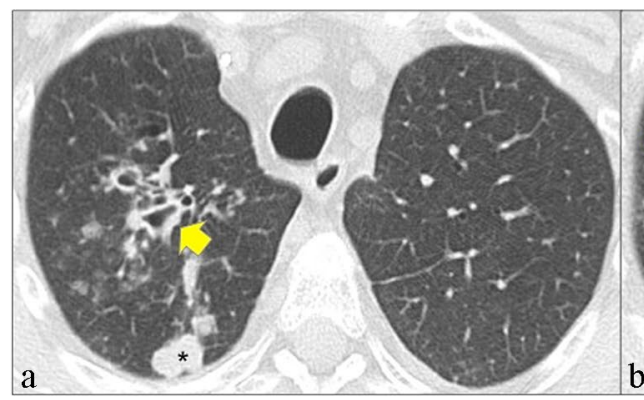

Fig. 7 Cytomegalovirus (CMV) infection in 39-year-old man that occurred 3 months after bilateral lung transplant for cystic fibrosis. The axial CT images demonstrate usual CMV findings, such as con-

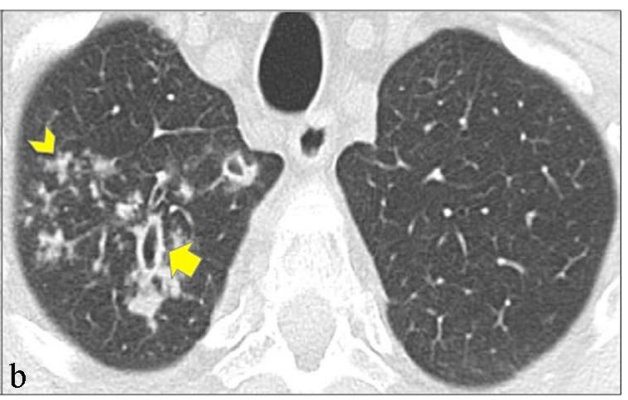

solidation (yellow asterisk in a), bronchiectasis (yellow arrows in a and $\mathbf{b}$ ) and tree-in-bud opacities (yellow arrowhead in $\mathbf{b}$ ) in the upper lobes

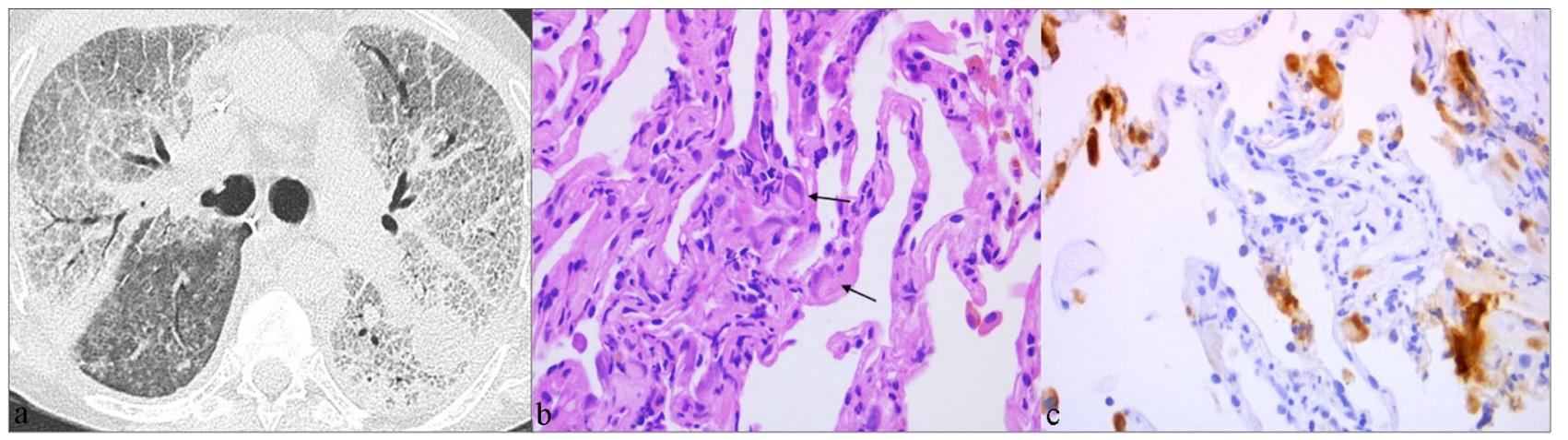

Fig. 8 Severe Cytomegalovirus pneumonia in a 35-year-old man who underwent bilateral lung transplant for idiopathic pulmonary fibrosis 2 months before showing a crazy paving pattern (i.e., diffuse groundglass opacifications with interolobular septal thickening) (a). The transbronchial biopsy showed a mild lymphocytic interstitial infiltrate and "atypical" intranuclear inclusion (arrow in b) of pneumocytes (b hematoxylin and eosin, original magnification $\times 400$ ). Immunohistochemistry confirmed the presence of CMV highlighting many other infected pneumocytes (c original magnification $\times 400$ ) 


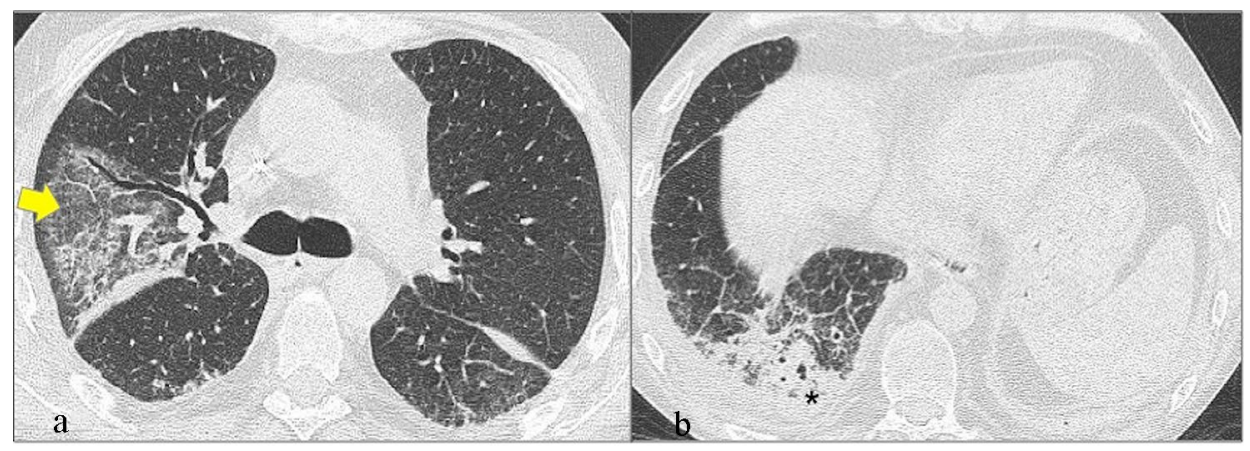

Fig. 9 Cytomegalovirus and Pseudomonas aeruginosa simultaneous infections in a 34-year-old man who underwent bilateral lung transplant for Langerhans cell histiocytosis 3 months before. The axial computed tomography images show the typical findings of the viral infection in the middle lobe (i.e., ground glass and interstitial thickening; yellow arrow in a), and of the bacterial infection in the right lower lobe (i.e., consolidation; black asterisk in b)

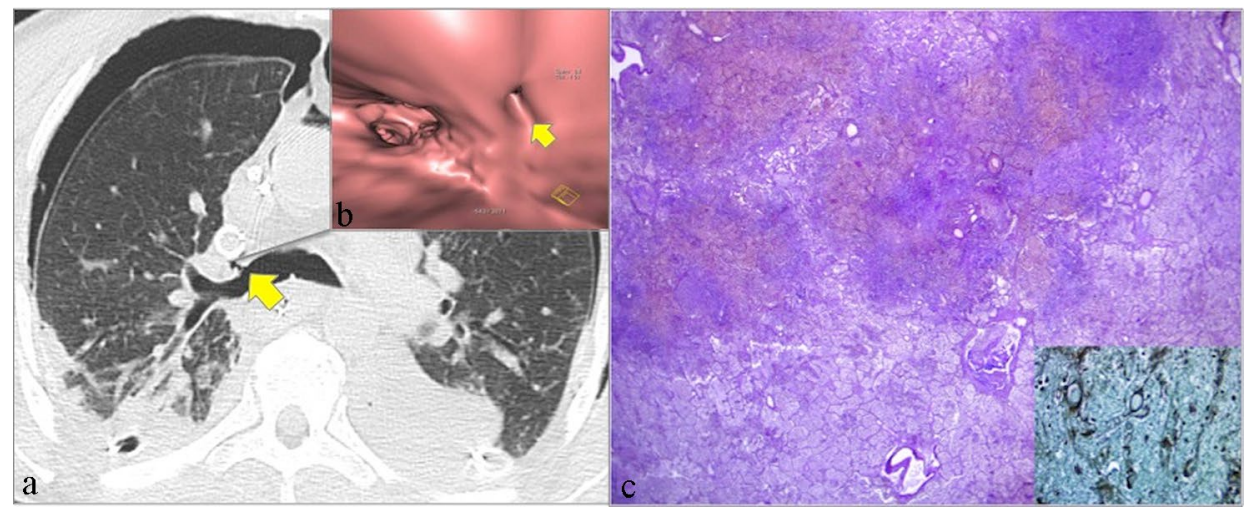

Fig. 10 Aspergillus pulmonary infection in a 40-year-old man 40 days after bilateral lung transplant for idiopathic pulmonary fibrosis. The axial computed tomography image (a) and the virtual bronchial endoscopy 3D-reconstruction (b) demonstrate well a bronchial fistula of the right main bronchus (yellow arrow in $\mathbf{a}$ and $\mathbf{b}$ ) due to necrotizing tracheobronchial aspergillosis (i.e., finding confirmed at autopsy). The autopsy specimen showed necrotizing pneumonia with several hyphae, morphologically compatible with Aspergillus spp. (c hematoxylin and eosin, original magnification $\times 12.5$; inset: Grocott's methenamine silver stain, original magnification $\times 400$ )
Fig. 11 Angioinvasive aspergillosis in a 41-year-old man who underwent bilateral lung transplant for cystic fibrosis 4 months before. Axial computed tomography image demonstrating a solitary mass in the middle lobe, which one year later evolved into a cavitary lesion (arrow in $\mathbf{a}$ and $\mathbf{b}$, respectively)

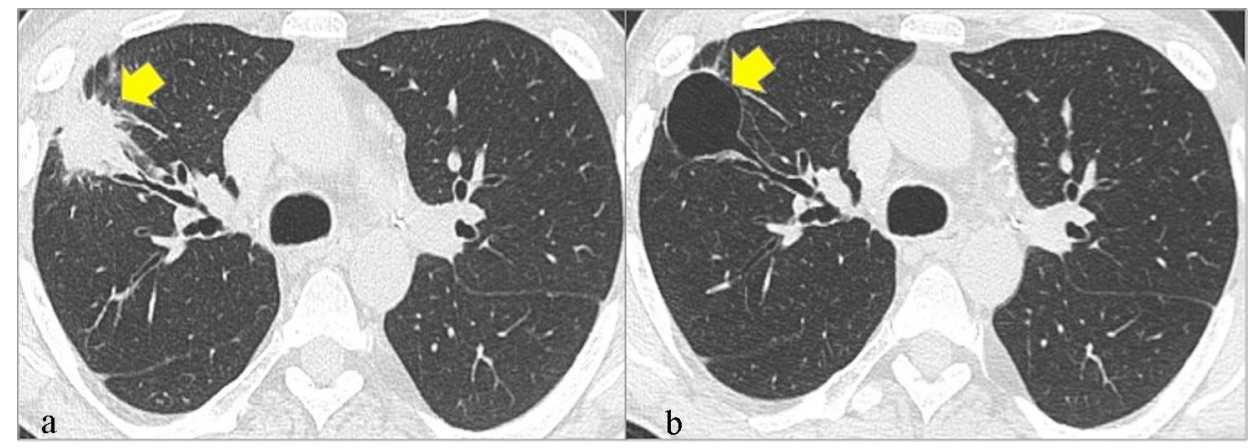




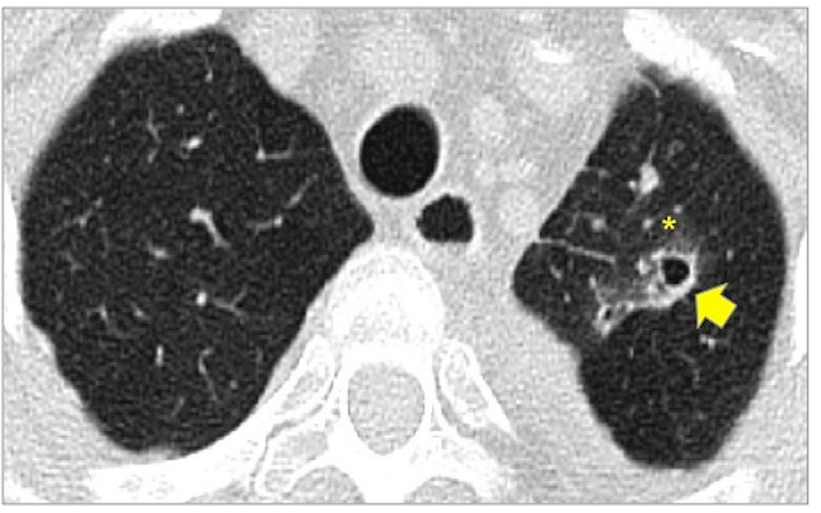

Fig. 12 Angioinvasive aspergillosis in 32-year-old woman who underwent bilateral lung transplant for lymphangioleiomyomatosis 2 months before. The axial computed tomography demonstrated an excavated nodule (yellow arrow) surrounded by a ground-glass halo (yellow asterisk) in the apicoposterior segment of the left upper lobe

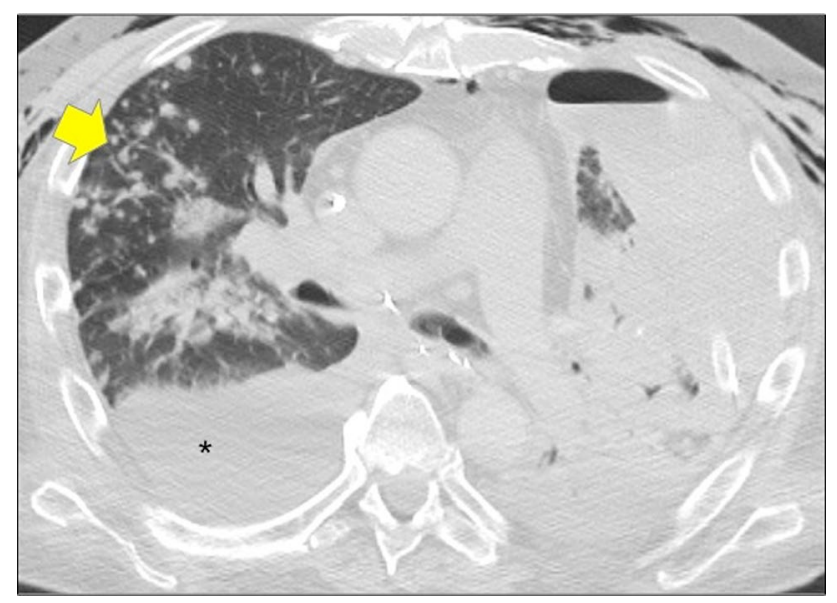

Fig. 13 Mycobacterium tuberculosis infection in a 57-year-old man six months after bilateral lung transplant for idiopathic pulmonary fibrosis showing miliary nodules (yellow arrow), and pleural effusion (black asterisk) infiltrates, consolidations, cavitations, interlobular septal and/or pleural thickenings, unilateral or bilateral pleural effusions, and mediastinal enlarged lymph nodes (Figs. 13 and 14) [1].

\section{Fungal infection}

Prior to the trimethoprim/sulfamethoxazole prophylaxis, Pneumocystis jirovecii pneumonia (PJP) was diagnosed in around $90 \%$ of the LT recipients and the first 6 months after the LT were at very high risk (i.e., highest peak between the second and sixth month). Nowadays, PJP is very uncommon during the first year after LT and it mainly occurs later on [16].

Typical PJP findings at CT are represented by bilateral ground-glass opacities with sub-pleural sparing and predilection for the upper lobes. Less frequently, focal areas of consolidation and thickening of the interlobular septa or diffuse micro-nodules are evident at imaging (Fig. 15). A cystic form of PJP may also occur and these patients carry a high risk of pneumothorax or pneumomediastinum [16].

\section{Conclusion}

Pulmonary infections are very common after LT and CT imaging plays a crucial role. Therefore, a deep knowledge of the typical CT findings caused by the most common different pathogens and their usual timing of colonization are essential to support the clinicians in the diagnostic and therapeutic process. 


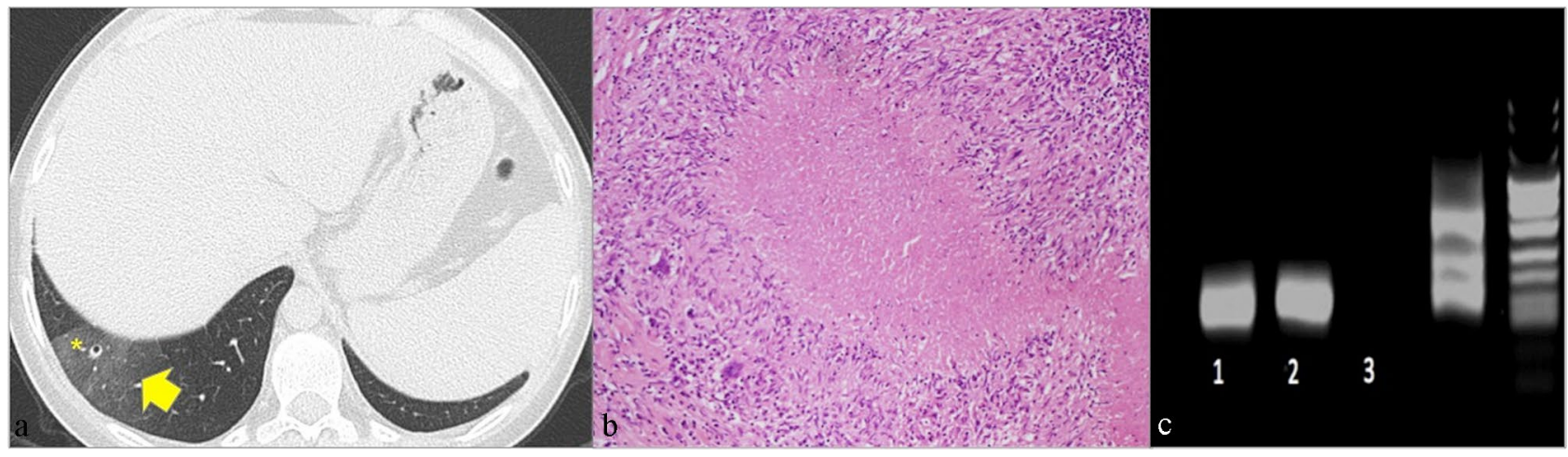

Fig. 14 Atypical Mycobacterial infection in a 20-year-old woman five months after bilateral lung transplant for cystic fibrosis. The axial computed tomography image demonstrates a ground-glass area in the right lower lobe (yellow arrow in a) and a small excavated lesion (yellow asterisk in a). The lung specimen showed a necrotizing granuloma with multinucleated giant cells (b hematoxylin and eosin, original magnification $\times 100$ ). Nested PCR for mycobacteria (using primers for IS6110 and hsp65) showed a strong positivity for hps65. Gene sequencing characterized the PCR results as a Mycobacterium simiae (c 1: lung; 2: atypical mycobacterial positive control; 3: negative control)

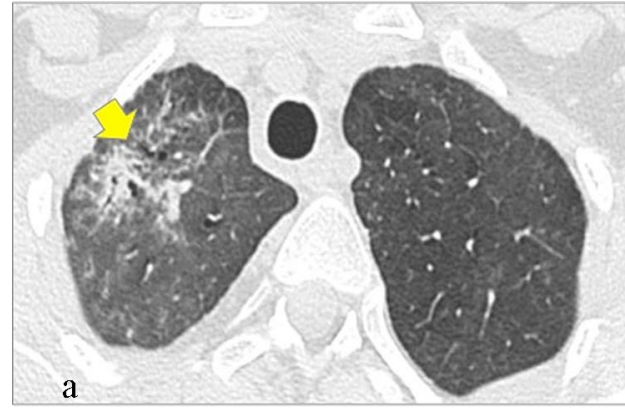

Fig. 15 Atypical computed tomography findings of Pneumocystis jirovecii in an 18-year-old woman receiving trimethoprim/sulfamethoxazole prophylaxis, who underwent bilateral lung transplant for cystic fibrosis one-and-a-half year before: patchy area of consolida-

Author contributions Conceptualization: CG; Methodology: CG, RM; Resources: FC, RP, EB, ML; Writing original draft: CG; Writing review and editing: FC, RP, RM, FR; Visualization: GR, LM, FP, FC; Supervision: CG, FR.

\section{Declarations}

Conflict of interest The authors have no conflicts of interest to declare that are relevant to the content of this article.

Ethical Standards All procedures performed in studies involving human participants were in accordance with the ethical standards of the institutional and/or national research committee and with the 1964 Helsinki Declaration and its later amendments or comparable ethical standards. The authors did not receive support from any organization for the submitted work

Open Access This article is licensed under a Creative Commons Attribution 4.0 International License, which permits use, sharing, adaptation, distribution and reproduction in any medium or format, as long

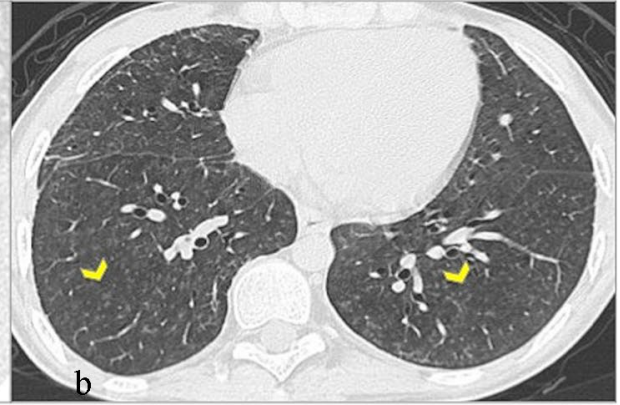

tion in the apical segment of the right upper lobe (yellow arrow in a) and bilateral diffuse centrilobular nodules in the lower lobes (arrowhead in b)

as you give appropriate credit to the original author(s) and the source, provide a link to the Creative Commons licence, and indicate if changes were made. The images or other third party material in this article are included in the article's Creative Commons licence, unless indicated otherwise in a credit line to the material. If material is not included in the article's Creative Commons licence and your intended use is not permitted by statutory regulation or exceeds the permitted use, you will need to obtain permission directly from the copyright holder. To view a copy of this licence, visit http://creativecommons.org/licenses/by/4.0/.

\section{References}

1. Krishnam MS, Suh RD, Tomasian A, Goldin JG, Lai C, Brown K, Batra P, Aberle DR (2007) Postoperative complications of lung transplantation: radiologic findings along a time continuum. Radiographics 27:957-974. https://doi.org/10.1148/rg.274065141

2. Amadi CC, Galizia MS, Mortani Barbosa EJ (2019) Imaging evaluation of lung transplantation patients a time and etiology-based approach to high-resolution computed tomography interpretation. 
J Thorac Imaging 34:299-312. https://doi.org/10.1097/RTI.00000 00000000419

3. Habre C, Soccal PM, Triponez F, Aubert JD, Krueger T, Martin SP, Gariani J, Pache JC, Lador F, Montet X, Hachulla AL (2018) Radiological findings of complications after lung transplantation. Insights Imaging 9:709-719. https://doi.org/10.1007/ s13244-018-0647-9

4. Kim SJ, Azour L, Hutchinson BD, Shirsat H, Zhou F, Narula N, Moreira AL, Angel L, Ko JP, Moore WH (2021) Imaging course of lung transplantation: from patient selection to postoperative complications. Radiographics 41:1043-1063. https://doi.org/10. $1148 /$ rg. 2021200173

5. Ward S, Müller NL (2000) Pulmonary complications following lung transplantation. Clin Radiol 55:332-339. https://doi.org/10. 1053/crad.2000.0439

6. Fishman JA (2017) Infection in organ transplantation. Am J Transplant 17:856-879. https://doi.org/10.1111/ajt.14208

7. Davda S, Kowa XY, Aziz Z, Ellis S, Cheasty E, Cappocci S, Balan A (2018) The development of pulmonary aspergillosis and its histologic, clinical, and radiologic manifestations. Clin Radiol 73:913-921. https://doi.org/10.1016/j.crad.2018.06.017

8. Collins J, Müller NL, Kazerooni EA, Paciocco G (2000) CT findings of pneumonia after lung transplantation. AJR Am J Roentgenol 175:811-818. https://doi.org/10.2214/ajr.175.3.1750811

9. Ng YL, Paul N, Patsios D, Walsham A, Chung TB, Keshavjee S, Weisbrod (2009) Imaging of lung transplantation: review. AJR Am J Roentgenol 192:S1-13. https://doi.org/10.2214/AJR.07.7061

10. Bassiri AG, Girgis RE, Theodore J (1996) Actinomyces odontolyticus thoracopulmonary infections. Two cases in lung and heartlung transplant recipients and a review of the literature. Chest 109:1109-1111. https://doi.org/10.1378/chest.109.4.1109

11. Remund KF, Best M, Egan JJ (2009) Infections relevant to lung transplantation. Proc Am Thorac Soc 6:94-100. https://doi.org/ 10.1513/pats.200809-113GO

12. Clark NM, Weigt SS, Fishbein MC, Kubak B, Belperio JA, Lynch JP (2018) Fungal infections complicating lung transplantation.
Semin Respir Crit Care Med 39:227-254. https://doi.org/10. 1055/s-0037-1617443

13. Baker AW, Maziarz EK, Arnold CJ, Johnson MD, Workman AD, Reynolds JM, Perfect JR, Alexander BD (2020) Invasive fungal infection after lung transplantation: epidemiology in the setting of antifungal prophylaxis. Clin Infect Dis 70:30-39. https://doi. org/10.1093/cid/ciz156

14. Koo HJ, Lim S, Choe J, Choi SH, Sung H, Do KH (2018) Radiographic and CT features of viral pneumonia. Radiographics 38:719-739. https://doi.org/10.1148/rg.2018170048

15. Almaghrabi RS, Omrani AS, Memish ZA (2017) Cytomegalovirus infection in lung transplant recipients. Expert Rev Respir Med 11:377-383. https://doi.org/10.1080/17476348.2017.1317596

16. Tejwani V, Panchabhai TS, Kotloff RM, Mehta AC (2016) Complications of lung transplantation: a roentgenographic perspective. Chest 149:1535-1545. https://doi.org/10.1016/j.chest.2015. 12.019

17. Franquet $\mathrm{T}$ (2011) Imaging of pulmonary viral pneumonia. Radiology 260:18-39. https://doi.org/10.1148/radiol.11092149

18. Kosmidis C, Denning DW (2015) The clinical spectrum of pulmonary aspergillosis. Thorax 70:270-277. https://doi.org/10.1136/ thoraxjnl-2014-206291

19. Erasmus JJ, McAdams HP, Tapson VF, Murray JG, Davis RD (1997) Radiologic issues in lung transplantation for end-stage pulmonary disease. AJR Am J Roentgenol 169:69-78

20. Dorman S, Subramanian A (2009) Nontuberculous mycobacteria in solid organ transplant recipients. Am J Transplant 4:S63-69. https://doi.org/10.1111/j.1600-6143.2009.02895.x

21. Ose N, Minami M, Funaki S, Kanou T, Kanzaki R, Shintani Y (2019) Nontuberculous mycobacterial infection after lung transplantation: a report of four cases. Surg Case Rep 5:11. https://doi. org/10.1186/s40792-019-0565-1

Publisher's Note Springer Nature remains neutral with regard to jurisdictional claims in published maps and institutional affiliations. 